\title{
Extração da catarata pela técnica de facoemulsificação em pacientes com uveíte
}

\author{
Cataract extraction using the phacoemulsification technique in patients with uveitis
}

\author{
Cláudio de Lima Yamane ${ }^{1}$ \\ Raul N. G. Vianna ${ }^{2}$ \\ Gilberto Perez Cardoso ${ }^{3}$ \\ Jean Deschênes ${ }^{4}$ \\ Mig'uel N. Burnier Jr. 5
}

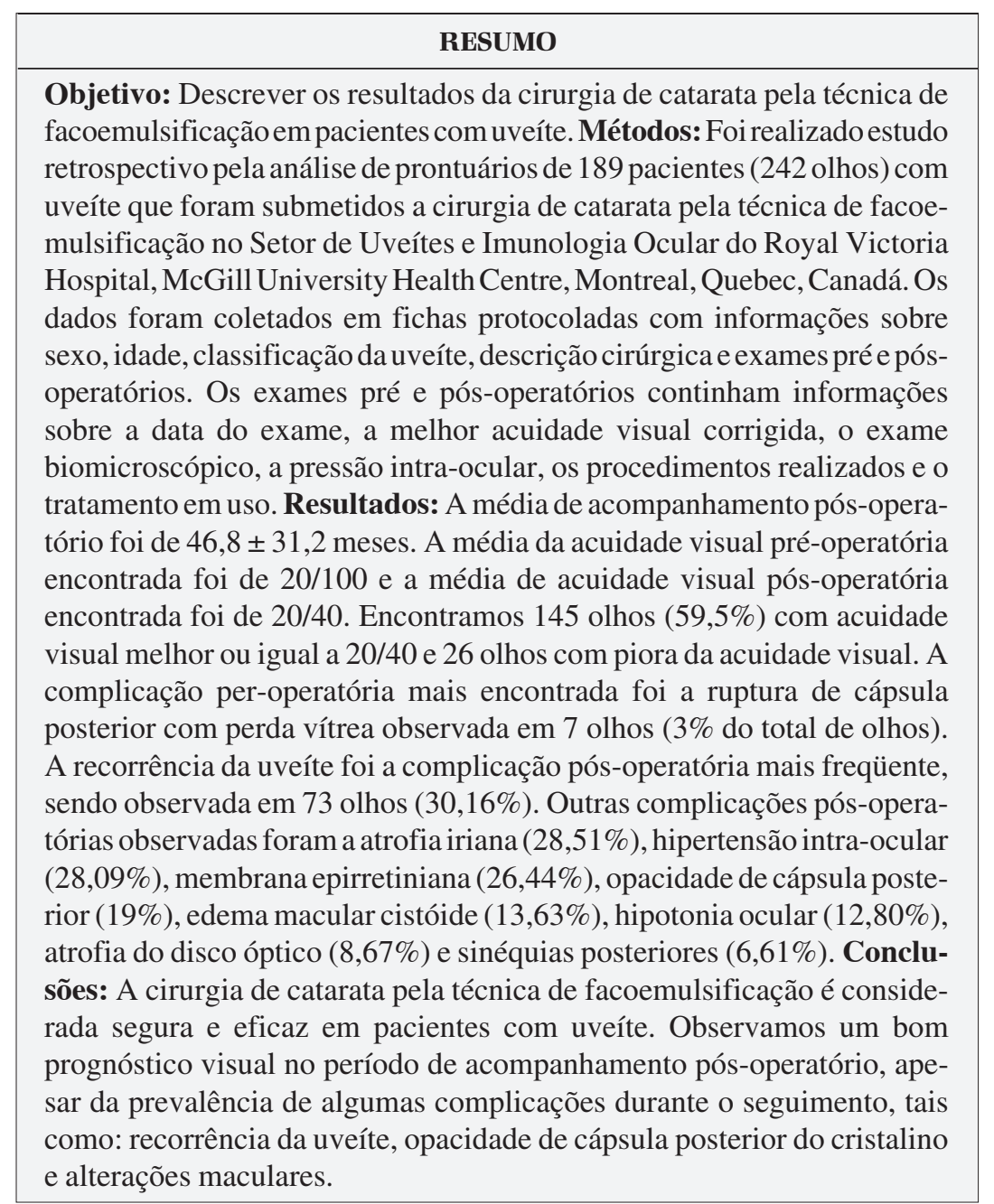

Descritores: Extração de catarata/métodos; Facoemulsificação; Implante de lente intraocular; Uveíte

\section{INTRODUÇÃO}

O desenvolvimento de catarata em pacientes com uveíte é comum. A prática da cirurgia de catarata pela técnica extracapsular com implante de lente intra-ocular (LIO) de câmara posterior nestes pacientes tem sido geralmente aceita como segura e como opção terapêutica efetiva ${ }^{(1)}$. No entanto, em pacientes com uveíte seria desejável uma técnica cirúrgica que desencadeasse a menor reação inflamatória possível. Autores mostram que 
em pacientes sem uveíte a cirurgia de catarata pela técnica de facoemulsificação pode induzir menos processo inflamatório do que a cirurgia extracapsular. Contudo, são poucas as publicações científicas que descreveram os resultados da técnica de facoemulsificação em pacientes com uveíte ${ }^{(2)}$.

No único artigo que relatou os resultados da cirurgia da catarata em pacientes com uveíte, utilizando exclusivamente a técnica de facoemulsificação, Estafanous et al analisaram os prontuários de 32 pacientes ( 39 olhos) e avaliaram a acuidade visual no pós-operatório, bem como a freqüência de algumas complicações tais como a opacidade de cápsula posterior, a recorrência da uveíte, o edema macular cistóide, a membrana epirretiniana e as sinéquias posteriores ${ }^{(2)}$.

As complicações descritas acima são, algumas vezes, resultados de inflamação e podem ocorrer menos freqüentemente em pacientes submetidos à cirurgia de facoemulsificação. Entretanto, para uma análise mais significativa seria desejável uma casuística maior de pacientes. O objetivo do presente trabalho foi descrever as complicações per e pós-operatórias da cirurgia da catarata pela técnica da facoemulsificação em 189 pacientes (242 olhos) com uveíte, bem como avaliar a acuidade visual desses pacientes durante o acompanhamento pós-operatório. De nosso conhecimento, esta é a maior casuística da literatura até o momento.

\section{MÉTODOS}

Foi realizado um estudo retrospectivo pela análise de prontuários do Setor de Uveítes e Imunologia Ocular da Universidade McGill, em Montreal, Quebec, Canadá.

Os dados foram coletados em fichas protocoladas com informação sobre sexo, idade, classificação da uveíte, descrição cirúrgica e exames pré e pós-operatórios. Os exames pré e pós-operatórios continham informações sobre a melhor acuidade visual corrigida, a pressão intra-ocular, o exame biomicroscópico, os procedimentos realizados e o tratamento em uso. Pacientes que apresentaram prontuários com dados incompletos, bem como aqueles submetidos a cirurgia da catarata pela técnica extracapsular foram excluídos do estudo.

$\mathrm{Na}$ análise da acuidade visual, foi utilizado o padrão da tabela de Snellen. Seguindo os critérios de Holladay ${ }^{(3)}$, consideramos todos os casos de acuidade visual de conta-dedos como acuidade visual de 20/2000, todos os casos de acuidade visual de movimento de mão como acuidade visual de 20/ 20000. Excluímos da análise uni ou bivariada da acuidade visual todos os casos de percepção luminosa e ausência de percepção luminosa.

De acordo com o Standardization of Uveitis Nomenclature (SUN) Working Group, utilizamos a acuidade visual como variável mais importante para análise de acompanhamento a longo prazo em pacientes com uveíte; classificamos as uveítes anatomicamente em anteriores, intermediárias, posteriores e difusas (ou panuveítes); definimos recorrência da uveíte como episódios repetidos separados por períodos de inatividade sem tratamento por período maior ou igual a três meses; e hipertensão intra-ocular como duas medidas de pressão intraocular maiores ou iguais a $21 \mathrm{mmHg}$ ou uma maior do que 30 $\mathrm{mmHg}^{(4)}$. Hipotonia ocular foi definida como pressão intraocular menor do que $6,5 \mathrm{mmHg}^{(5)}$.

No acompanhamento pós-operatório, foram registradas a primeira consulta pós-operatória que ocorria no primeiro dia após a cirurgia e a última consulta de acompanhamento de cada paciente. Além disso, foram colhidas todas as consultas intermediárias do pós-operatório em que ocorria alguma alteração do exame do paciente.

Os dados foram transferidos para o Statistical Package for the Social Science ${ }^{\circledR}$ (SPSS versão 11) e posteriormente foi realizada uma análise univariada e bivariada para selecionarmos quais as variáveis independentes que melhor se correlacionam com o evento final (eficácia da cirurgia).

\section{RESULTADOS}

Foram estudados 189 pacientes ( 242 olhos) com média de idade de 51,7 $\pm 15,8$ anos (mediana de 52,4 anos; idade mínima de 15,5 e máxima de 85,6$)$. Foram encontrados $80(80 / 189=$ $42,3 \%)$ pacientes do sexo masculino e $109(57,7 \%)$ do sexo feminino.

O tempo médio de acompanhamento entre a cirurgia e a última consulta foi de 3,9 $\pm 2,6$ anos (mediana de 3,4 anos).

Quanto à classificação anatômica das uveítes, verificamos 165 casos de uveíte anterior, 17 de intermediária, 17 de posterior e 43 de difusa.

Quanto à classificação etiológica das uveítes, verificamos um total de 248 casos, ocorrendo dois tipos etiológicos diferentes no mesmo paciente em 6 casos. As etiologias se distribuíram da seguinte maneira: idiopática (105 casos), iridociclite heterocrômica de Fuchs (34 casos), Herpes simplex (18 casos), sarcoidose (14 casos), pars planitis (10 casos), artrite reumatóide juvenil (10 casos), esclerose múltipla (6 casos), citomegalovírus (06 casos), espondilite anquilosante (5 casos), doença de Behçet (5 casos), coroidite multifocal (4 casos), coroidite multifocal com panuveíte (4 casos), coriorretinopatia de Birdshot (3 casos), síndrome de Vogt-Koyanagi-Harada (3 casos), artrite psoriática (2 casos), doença de Crohn (2 casos), retinocoroidite por toxoplasmose ( 2 casos), tuberculose ( 2 casos), HIV ( 2 casos), necrose retiniana aguda ( 2 casos), herpes zoster (1 caso), síndrome de Reiter (1 caso), granulomatose de Wegener (1 caso), coroidite serpiginosa (1 caso), oftalmia simpática (1 caso), síndrome de Posner-Schlossman (1 caso), glaucoma facolítico (1 caso), doença de Eales (1 caso), lupus eritematoso sistêmico (1 caso). Dos 6 casos com dois tipos etiológicos de uveíte, observamos um de iridociclite heterocrômica de Fuchs associado à síndrome de Posner-Schlossman, dois casos de iridociclite heterocrômica de Fuchs associado a coroidite multifocal com panuveíte, dois casos de retinite por citomegalovírus associado ao HIV e um caso de doença de Behçet associado a ceratouveíte por herpes simples. 
Quanto às lentes intra-oculares, verificamos lentes de três materiais e 14 modelos diferentes. Os três materiais utilizados foram o polimetilmetacrilato (PMMA), o PMMA modificado com superfície heparinizada (HSM-PMMA) e o acrílico. Os modelos de PMMA foram: 720A (Pharmacia), 6741B (Bausch \& Lomb), 8191B (Bausch \& Lomb), 8190B (Bausch \& Lomb), U85JS (Bausch \& Lomb), MC50BD (Alcon), MZ20BD (Alcon) e MZ40BD (Alcon); os modelos de PMMA com superfície modificada heparinizada foram: 722C (Pharmacia), 809C (Pharmacia), 812C (Pharmacia); e os modelos de acrílico foram: MA30BA (Alcon), MA60BM (Alcon) e SA60AT (Alcon).

Dos 242 olhos submetidos a cirurgia de catarata pela técnica de facoemulsificação, verificamos 223 olhos com implante da lente intra-ocular e 19 sem seu implante.

Dos 223 olhos em que houve implante de LIO, verificamos 141 com lente de PMMA, 60 com LIO acrílica e 22 com LIO heparinizada (HSM-PMMA). Dos casos com implante da LIO, 219 evoluíram no período pós-operatório sem necessidade de seu explante e 4 evoluíram com necessidade de explante. Estes olhos com LIO explantada tiveram como etiologias associadas a uveíte idiopática (3 olhos) e a pars planitis (um olho), e todos os pacientes foram do sexo feminino.

Dos olhos em que não houve implante da LIO, as etiologias associadas foram uveíte idiopática ( 8 olhos), artrite reumatóide juvenil (5 olhos), doença de Behçet (2 olhos), sarcoidose (1 olho), necrose retiniana aguda (1 olho), citomegalovirus associado ao HIV (1 olho) e oftalmia simpática (1 olho). Destes, 17 olhos evoluíram sem complicações per-operatórias e dois evoluíram com complicações per-operatórias. As causas para o não implante da LIO foram: uveíte grave (10 olhos), artrite reumatóide juvenil (5 olhos), complicação per-operatória (2 olhos), edema de córnea pré-operatório (1 olho) e objetivo cirúrgico apenas para observação do fundo de olho (1 olho).

As alterações biomicroscópicas apresentadas no exame pré-operatório estão descritas na tabela 1 .

Foram encontrados 14 casos com complicações per-operatórias, dentre eles um caso de LIO no sulco ciliar, 4 casos de LIO no sulco ciliar e rotura de cápsula posterior, 5 casos de LIO no sulco ciliar, rotura de cápsula posterior e perda vítrea, 2 casos de rotura de cápsula posterior e perda vítrea, 1 caso de LIO na câmara anterior, rotura de cápsula posterior e perda vítrea e 1 caso de restos corticais e hemorragia expulsiva.

As alterações biomicroscópicas apresentadas no exame pós-operatório estão descritas na tabela 2 .

A acuidade visual média do pré-operatório foi de 20/100, da última consulta de acompanhamento pós-operatório foi de 20/40 e do tempo médio de acompanhamento pós-operatório foi de 20/40. Isto resultou em uma melhora da acuidade visual de 5 linhas de Snellen. Observamos que 146 olhos alcançaram acuidade visual melhor ou igual a 20/40 quando avaliamos a última consulta de acompanhamento pós-operatório e 145 $(59,90 \%)$ quando avaliamos o tempo médio de acompanhamento pós-operatório (Gráfico 1).

Encontramos 20 olhos (8,8\%) que evoluíram com baixa da

\begin{tabular}{|lcc|}
\hline \multicolumn{3}{|c|}{ Tabela 1. Alterações pré-operatórias da amostra } \\
\hline Alterações pré-operatórias & $\begin{array}{c}\text { № de } \\
\text { alterações }\end{array}$ & $\begin{array}{c}\% \text { de } \\
\text { alterações }\end{array}$ \\
\hline Sinéquias posteriores & 93 & $38,43 \%$ \\
Atrofia iriana & 47 & $19,42 \%$ \\
Membrana epirretiniana & 28 & $11,57 \%$ \\
Edema macular cistóide & 27 & $11,15 \%$ \\
Cicatrizes corneanas & 14 & $5,78 \%$ \\
Ceratopatia em faixa & 8 & $3,30 \%$ \\
Atrofia de disco óptico & 5 & $2,06 \%$ \\
Rubeosis iridis & 4 & $1,65 \%$ \\
Edema de disco óptico & 4 & $1,65 \%$ \\
Sinéquias anteriores & 3 & $1,24 \%$ \\
Opacidade vítrea & 3 & $1.24 \%$ \\
Opacidade de cápsula posterior & 2 & $0,82 \%$ \\
Opacidade de cápsula anterior & 2 & $0,82 \%$ \\
Edema corneano crônico & 1 & $0,41 \%$ \\
\hline
\end{tabular}

acuidade visual durante o acompanhamento pós-operatório, quando comparamos a consulta pré-operatória com a última consulta de acompanhamento pós-operatório, num total de 226 dos 242 olhos. Destes, 16 olhos foram excluídos da análise devido à acuidade visual de amaurose ou percepção luminosa; e 26 olhos $(11,4 \%)$ evoluíram com baixa da acuidade visual quando comparamos a consulta pré-operatória com o tempo médio de acompanhamento pós-operatório, num total de 228 dos 242 olhos. Destes, 14 olhos foram excluídos da análise devido à acuidade visual de amaurose ou percepção luminosa.

Dos procedimentos cirúrgicos realizados, verificamos 64 olhos submetidos a capsulotomia posterior por $\mathrm{Nd}$ :Yag laser; 14 a trabeculectomia; 9 a vitrectomia total; 8 a vitrectomia anterior; 6 a válvula de Ahmed; 2 a evisceração; 1 a retinopexia; e 1 a enucleação.

\section{DISCUSSÃO}

O objetivo do presente trabalho foi descrever os resultados da cirurgia de extração de catarata através da técnica de facoemulsificação em pacientes com uveíte. Por isso, para tornar o estudo viável, buscamos um desenho de pesquisa retrospectivo, o estudo bidirecional. Isto permitiu que conseguíssemos uma amostra numericamente significativa. Estudos anteriores seguiram um desenho epidemiológico retrospectivo conforme nosso estudo, exceto um, prospectivo.

Em nosso estudo analisamos uma amostra de 189 pacientes com uveíte (242 olhos), submetidos a cirurgia da catarata pela técnica da facoemulsificação com implante de LIO de câmara posterior. Comparando nossa amostra com estudos anteriores, podemos perceber que conseguimos um número bastante significativo, principalmente por estarmos analisando apenas as cirurgias através da técnica de facoemulsificação ${ }^{(1-2,6-7)}$. De nosso conhecimento, esta é a maior casuística da literatura até o momento. 


\begin{tabular}{|c|c|c|}
\hline Alterações pós-operatórias & $\begin{array}{c}\text { № de } \\
\text { alterações }\end{array}$ & $\begin{array}{c}\% \text { de } \\
\text { alterações }\end{array}$ \\
\hline Recorrência da uveíte & 73 & $30,16 \%$ \\
\hline Atrofia iriana & 69 & $28,51 \%$ \\
\hline Hipertensão intra-ocular & 68 & $28,09 \%$ \\
\hline Membrana epirretiniana & 64 & $26,44 \%$ \\
\hline Opacidade de cápsula posterior & 46 & $19,00 \%$ \\
\hline Edema macular cistóide & 33 & $13,63 \%$ \\
\hline Hipotensão intra-ocular & 31 & $12,80 \%$ \\
\hline Atrofia de disco óptico & 21 & $8,67 \%$ \\
\hline Sinéquias posteriores & 16 & $6,61 \%$ \\
\hline Opacidade vítrea & 16 & $6,61 \%$ \\
\hline Cicatrizes corneanas & 15 & $6,19 \%$ \\
\hline Fibrina na câmara anterior & 9 & $3,72 \%$ \\
\hline Rubeosis iridis & 9 & $3,72 \%$ \\
\hline Ceratopatia em faixa & 8 & $3,30 \%$ \\
\hline Edema corneano crônico & 5 & $2,06 \%$ \\
\hline Neovasos corneanos & 5 & $2,06 \%$ \\
\hline Explante da LIO & 4 & $1,65 \%$ \\
\hline Ceratopatia bolhosa & 3 & $1,24 \%$ \\
\hline Hifema & 3 & $1,24 \%$ \\
\hline Vítreo na câmara anterior & 3 & $1,24 \%$ \\
\hline Sinéquias anteriores & 3 & $1,24 \%$ \\
\hline Depósito na LIO & 3 & $1,24 \%$ \\
\hline Deslocamento da LIO & 3 & $1,24 \%$ \\
\hline Hemorragia vítrea & 3 & $1,24 \%$ \\
\hline Buraco macular & 3 & $1,24 \%$ \\
\hline Edema de disco óptico & 3 & $1,24 \%$ \\
\hline Phthisis bulbi & 3 & $1,24 \%$ \\
\hline Córnea transplantada & 2 & $0,82 \%$ \\
\hline Hipópio & 2 & $0,82 \%$ \\
\hline Opacidade de cápsula anterior & 2 & $0,82 \%$ \\
\hline Restos corticais & 2 & $0,82 \%$ \\
\hline Captura da LIO & 2 & $0,82 \%$ \\
\hline Olho eviscerado & 2 & $0,82 \%$ \\
\hline Perfuração corneana & 1 & $0,41 \%$ \\
\hline Descolamento de retina & 1 & $0,41 \%$ \\
\hline Descolamento de coróide & 1 & $0,41 \%$ \\
\hline Olho enucleado & 1 & $0,41 \%$ \\
\hline $\mathrm{LIO}=$ lente intra-ocular & & \\
\hline
\end{tabular}

A média de idade de nosso estudo foi de 51,7 $\pm 15,8$ anos (mediana de 52,4 anos; idade mínima de 15,5 e máxima de 85,6) e está compatível com a média de idade da literatura ${ }^{(2,6-7)}$.

Verificamos que 80 pacientes $(42,3 \%)$ eram do sexo masculino e $109(57,7 \%)$ do sexo feminino. O gênero em nosso estudo está compatível com a literatura, onde encontramos uma discreta prevalência do sexo feminino ${ }^{(1-2,6-67)}$.

Em nosso estudo, o tempo médio de acompanhamento entre a data da cirurgia e a última consulta pós-operatória foi de 3,9 \pm 2,6 anos (mediana 3,4 anos). Comparando com a literatura, percebemos que nosso tempo médio de acompanhamento pósoperatório (40,8 meses) foi bastante significativo ${ }^{(2,7)}$.

Quanto à classificação anatômica dos tipos de uveíte, encontramos $165(68,18 \%)$ olhos com uveíte anterior, 17 (7\%)

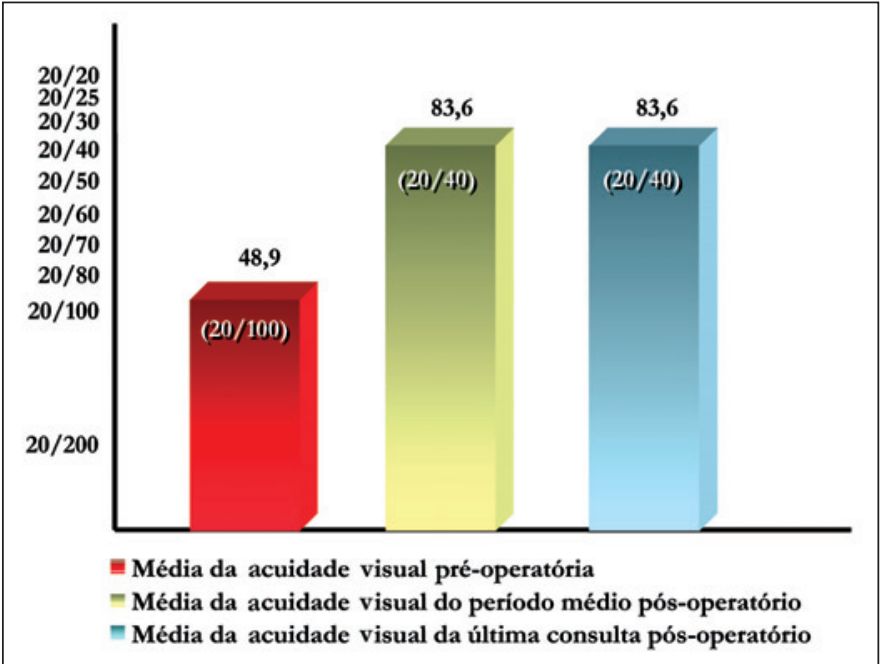

Gráfico 1 - Médias da acuidade visual pré-operatória, do período médio pós-operatório e da última consulta pós-operatório

com intermediária, 17 (7\%) com posterior e 43 (17,76\%) com difusa. A análise da classificação anatômica dos vários tipos de uveíte ficou prejudicada pelos diferentes sistemas de classificação adotados em cada trabalho ${ }^{(1-2,6-7)}$. O "The SUN Working Group" preconiza que as uveítes sejam classificadas em anterior, intermediária, posterior e panuveíte de acordo com o sítio primário de inflamação ${ }^{(4)}$. Apesar desta tentativa de padronização ter sido publicada em 2005, o Setor de Uveítes e Imunologia Ocular do Royal Victoria Hospital, da Universidade McGill, já seguia esta classificação.

Quanto à classificação etiológica das uveítes, os três tipos mais frequientes que encontramos foram uveíte idiopática (105 casos; 42,33\% dos casos), iridociclite heterocrômica de Fuchs (34 casos; $13,70 \%$ dos casos) e uveíte por herpes simples (18 casos; 7,25\% dos casos). Em todos os casos descritos previamente na literatura, a uveíte idiopática foi a mais preponderante, sendo compatível também com nosso estudo ${ }^{(1-2,7)}$.

Dos nossos 242 casos de cirurgia de catarata pela técnica de facoemulsificação, verificamos 223 casos com implante da LIO e 19 casos sem seu implante.

Dos 223 olhos em que houve implante de LIO, obtivemos $141(63,22 \%)$ olhos com lente de polimetilmetacrilato (PMMA), $60(26,90 \%)$ com acrílica e $22(09,86 \%)$ com lente de PMMA modificada com superfície heparinizada (HSM-PMMA). Dos estudos anteriores, o primeiro utilizou dois tipos de materiais para lentes intra-oculares: lente de PMMA isolado em 26 olhos e lente de PMMA associada a alças de prolene em 10 olhos; o segundo utilizou apenas lentes intra-oculares de PMMA modificado de superfície heparinizada; e o terceiro, apenas lentes intra-oculares de PMMA em todos os $\operatorname{casos}^{(1,6-7)}$. Apenas um estudo apresentou implante apenas lentes acrílicas em todos os $\operatorname{casos}^{(2)}$. Verificou-se que o uso de lentes intra-oculares dobráveis em olhos com uveíte é seguro, mas o biomaterial ótimo ainda não foi encontrado ${ }^{(8)}$.

Dos casos com implante da LIO, 219 evoluíram no período 
pós-operatório sem necessidade de seu explante e 4 evoluíram com necessidade de explante. Estes casos explantados tiveram como etiologias associadas a uveíte idiopática (3 casos) e a pars planitis (1 caso), e todos foram do sexo feminino. Observou-se em uma série de casos de pacientes com uveíte e catarata que foram submetidos a extração da catarata com implante de LIO de câmara posterior e que num segundo momento foram submetidos ao explante das lentes devido ao dano intra-ocular progressivo pela reação inflamatória. De fato, a remoção da LIO pode resgatar uma visão útil para os pacientes que continuam a exibir complicações secundárias a uveíte após a cirurgia, evitando danos irreversíveis à mácula e ao nervo óptico ${ }^{(9)}$.

Em nosso estudo, dos casos em que não houve implante da LIO, as etiologias associadas foram uveíte idiopática ( 8 olhos), artrite reumatóide juvenil (5 olhos), doença de Behçet (2 olhos), sarcoidose (1 olho), necrose retiniana aguda (1 olho), citomegalovirus associado ao HIV (1 olho) e oftalmia simpática (1 olho). Destes, 17 evoluíram sem complicações per-operatórias e dois evoluíram com complicações per-operatórias. As causas para o não implante da LIO foram: uveíte grave (10 olhos), artrite reumatóide juvenil (5 olhos), complicação per-operatória (2 olhos), edema de córnea pré-operatório (1 olho) e objetivo cirúrgico apenas para observação do fundo de olho (1 olho). Não encontramos na literatura estudos que citassem, como objetivo, as causas de não implante de LIO em pacientes com uveíte submetidos à cirurgia de catarata.

$\mathrm{Na}$ análise do número de olhos com melhora da acuidade visual, verificamos que, dos 242 olhos estudados para avaliação da acuidade visual durante o período médio de acompanhamento pós-operatório, 14 olhos foram excluídos. Esta exclusão foi devido à impossibilidade de comparação da acuidade visual pré-operatória com a acuidade visual do período médio pós-operatório de acuidades visuais de percepção luminosa e ausência de percepção luminosa (amaurose). Assim, encontramos que, dos 228 olhos incluídos, 202 (88,60\%) apresentaram melhora da acuidade visual e $26(11,40 \%)$ apresentaram manutenção ou piora da acuidade visual. Dos estudos anteriores, observou-se, no primeiro, uma melhora da acuidade visual em $97 \%$ dos olhos; no segundo, melhora da acuidade visual em $96 \%$ dos olhos com uveíte anterior e em $81 \%$ dos olhos com uveíte posterior; e no terceiro, melhora da acuidade visual em $95 \%$ dos olhos ${ }^{(1-2,7)}$.

Nosso estudo mostrou que da amostra de 242 olhos, o número de olhos com acuidade visual melhor ou igual a 20/40 foi de 145 (59,9\%), com acuidade visual entre 20/40 e 20/2000 foi de $82(33,9 \%)$ e com acuidade visual de movimento de mãos, percepção luminosa e ausência de percepção luminosa foi de 15 $(6,20 \%)$. Dentro do grupo de olhos com ausência de percepção luminosa, estão dois olhos eviscerados e um olho enucleado.

Verificamos uma melhora de 5 linhas de Snellen, quando comparamos a média das acuidades visuais pré-operatórias com a média das acuidades visuais do período médio pós-operatório. A melhora de acuidade visual em linhas de Snellen de nosso trabalho é estatisticamente significativa ( $\mathrm{p}<0,0001)$, e além disso, está compatível com a literatura ${ }^{(2)}$.
As alterações pré-operatórias mais freqüentes foram: sinéquias posteriores (93 olhos), atrofia iriana (47 olhos), membrana epirretiniana (28 olhos) e edema macular cistóide (27 olhos) (Tabela 1).

Observamos em nosso estudo que dos 242 olhos operados, $14(5,78 \%)$ evoluíram com complicações per-operatórias. Dos olhos complicados, 5 apresentaram $(35,71 \%)$ rotura de cápsula posterior, perda vítrea e LIO no sulco ciliar; $4,(28,57 \%)$ rotura de cápsula posterior e LIO no sulco ciliar; 2 (14,28\%), rotura de cápsula posterior e perda vítrea sem implante de LIO; 1 (7,14\%), rotura de cápsula posterior, perda vítrea e implante de LIO na câmara anterior; 1 (7,14\%), implante de LIO no sulco ciliar; e 1 $(7,14 \%)$, permanência de restos corticais no saco capsular e hemorragia expulsiva. Provavelmente, devido ao tamanho da amostra deste estudo, obtivemos um maior número de complicações per-operatórias ${ }^{(2,7)}$.

As complicações pós-operatórias mais freqüentes que encontramos em nosso estudo foram recorrência da uveíte (73 olhos; 30,16\%), atrofia iriana (69 olhos; 28,51\%), hipertensão intra-ocular (68 olhos 28,09\%), membrana epirretiniana (64 olhos; $26,44 \%)$, opacidade de cápsula posterior do cristalino $(46 ; 19,00 \%)$ e edema macular cistóide $(33 ; 13,63 \%)$. (Tabela 2$)$

As complicações pós-operatórias não encontradas em estudos anteriores foram: atrofia iriana, atrofia do disco óptico, cicatrizes corneanas, fibrina na câmara anterior, ceratopatia em faixa, edema corneano crônico, neovasos corneanos, necessidade de explante da LIO, ceratopatia bolhosa, vítreo na câmara anterior, buraco macular, edema do disco óptico, phthisis bulbi, necessidade de transplante de córnea, hipópio, opacidade de cápsula anterior, restos corticais, necessidade de evisceração ocular, descolamento de coróide e necessidade de enucleação ocular ${ }^{(1-2,7)}$.

Nosso trabalho apresentou pontos positivos. O primeiro deles se refere ao tamanho da amostra. Nenhum dos trabalhos anteriormente referidos apresentou uma amostra tão significativa de olhos com uveíte submetidos a cirurgia de catarata quanto este. $\mathrm{O}$ segundo ponto positivo se refere à utilização apenas da técnica de facoemulsificação, pois os trabalhos anteriores mostraram resultados da cirurgia de catarata pela técnica de extração extracapsular ou pela alternância das técnicas de extração extracapsular e de facoemulsificação. Apenas um estudo prévio utilizou exclusivamente a facoemulsificação, porém analisou-se apenas 39 olhos, deixando muitas respostas a serem dadas em futuros trabalhos ${ }^{(1,6-7)}$. O terceiro ponto positivo foi a possibilidade de descrever complicações pós-operatórias relacionadas à cirurgia de facoemulsificação em pacientes com uveíte que ainda não foram descritas em estudos semelhantes.

Dentre as limitações do presente trabalho, destacamos a impossibilidade de um acompanhamento pós-operatório por períodos regulares. Isto tornou inviável uma análise comparativa de alterações pós-operatórias em períodos de tempo (recentes, intermediários e tardios).

Nosso estudo revelou que a cirurgia de catarata pela técnica de facoemulsificação com implante de LIO de câmara posterior é segura e eficaz. Entretanto, devemos ponderar restrições 
para o implante da LIO durante o planejamento cirúrgico. E nos casos de implante de LIO, durante o acompanhamento pós-operatório, devemos ponderar a possibilidade do explante da mesma.

\section{CONCLUSÕES}

As complicações per-operatórias, em ordem de freqüência, foram: LIO no sulco ciliar associada a rotura de cápsula posterior e perda vítrea; LIO no sulco ciliar associado a rotura de cápsula posterior sem perda vítrea; rotura de cápsula posterior e perda vítrea; LIO no sulco ciliar; LIO de câmara anterior, rotura de cápsula posterior e perda vítrea; e persistência de restos corticais associado a hemorragia expulsiva.

As complicações pós-operatórias encontradas foram: recorrência da uveíte, atrofia iriana, hipertensão intra-ocular, membrana epirretiniana, opacidade de cápsula posterior, edema macular cistóide, hipotensão intra-ocular, atrofia do disco óptico, sinéquias posteriores, opacidade vítrea, cicatrizes corneanas, fibrina na câmara anterior, rubeosis iridis, ceratopatia em faixa, edema corneano crônico, neovasos corneanos, explante da LIO, ceratopatia bolhosa, hifema, vítreo na câmara anterior, sinéquias anteriores, depósito na LIO, deslocamento da LIO, hemorragia vítrea, buraco macular, edema do disco óptico, phthisis bulbi, córnea transplantada, hipópio, opacidade de cápsula anterior, restos corticais, captura da LIO, necessidade de evisceração ocular, perfuração corneana, descolamento de retina, descolamento de coróide e necessidade de enucleação.

Verificamos no resultado pós-operatório uma melhora da média da acuidade visual em cinco linhas de Snellen quando comparada com a média da acuidade visual pré-operatória.

\section{ABSTRACT}

Purpose: To report outcomes of cataract extraction by phacoemulsification in patients with uveitis. Methods: We retrospectively reviewed the charts of 189 patients (242 eyes) with uveitis who underwent cataract extraction by phacoemulsification at the Uveitis and Ocular Immunology Unit of McGill University Health Centre, Montreal, Quebec, Canada. Results: Average follow-up was $46 \pm 31.2$ months. Average preoperative visual acuity was 20/100 and average postoperative visual acuity was 20/40. Hundred and forty-six eyes (59.9\%) attained visual acuity better than 20/40. Visual loss occurred in 26 eyes. The most common peroperative complication was posterior capsule rupture with vitreous loss seen in 7 eyes (3\%). Recurrence of uveitis was the most common postoperative complication seen in 73 eyes $(30.16 \%)$. Other postoperative complications included iris atrophy $(28.51 \%)$, ocular hypertension (28.09\%), epiretinal membrane $(26.44 \%)$, posterior capsule opacification (19\%), cystoid macular edema (13.63\%), ocular hypotony $(12.80 \%)$, optic disc atrophy $(8.67 \%)$ and posterior synechiae $(6.61 \%)$. Conclusions: Cataract extraction by phacoemulsification is safe in patients with uveitis. Successful visual results are observed in long-term follow-up despite the prevalence of recurrence of uveitis, posterior capsule opacification and macular abnormalities. To the best of our knowledge this is the largest series presented to date.

Keywords: Cataract extraction/methods; Phacoemulsification; Lens implantation, intraocular; Uveitis

\section{REFERÊNCIAS}

1. Krishna R, Meisler DM, Lowder CY, Estafanous M, Foster RE. Long-term follow-up of extracapsular cataract extraction and posterior chamber intraocular lens implantation in patients with uveitis. Ophthalmology. 1998;105(9):1765-9.

2. Estafanous MF, Lowder CY, Meisler DM, Chauhan R. Phacoemulsification cataract extraction and posterior chamber lens implantation in patients with uveitis. Am J Ophthalmol. 2001;131(5):620-5.

3. Holladay JT. Visual Acuity measurements. J Cataract Refract Surg. 2004;30 (2):287-90.

4. Jabs DA, Nussenblatt RB, Rosenbaum JT; Standardization of Uveitis Nomenclature (SUN) Working Group. Standardization of uveitis nomenclature for reporting clinical data. Results of the First International Workshop. Am J Ophthalmol. 2005;140(3):509-16.

5. Pederson JE. Ocular hypotony. In: Ritch R, Shields MB, Krupin T, editors. The glaucomas. 2nd ed. St Louis: Mosby; 1996. p.385.

6. Pivetti-Pezzi P, Accorinti M, La Cava M, Abdulaziz MA, Pantaleoni FB. Long-term follow-up of anterior uveitis after cataract extraction and intraocular lens implantation. J Cataract Refract Surg. 1999;25(11):1521-6.

7. Okhravi N, Lightman SL, Towler HM. Assessment of visual outcome after cataract surgery in patients with uveitis. Ophthalmology. 1999;106(4):710-22.

8. Rauz S, Stavrou P, Murray PI. Evaluation of foldable intraocular lenses in patients with uveitis. Ophthalmology. 2000;107(5):909-19.

9. Foster CS, Stavrou P, Zafirakis P, Rojas B, Tesavibul N, Baltatzis S. Intraocular lens removal from [corrected] patients with uveitis. Am J Ophthalmol. 1999;128(1):31-7. 